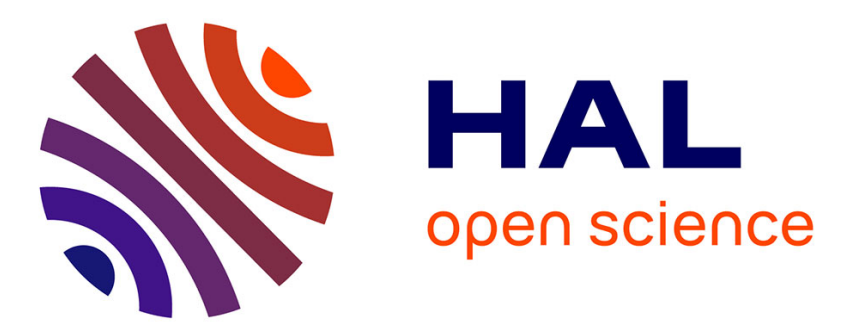

\title{
Inflation of elastomeric circular membranes using network constitutive equations
}

Erwan Verron, Gilles Marckmann

\section{To cite this version:}

Erwan Verron, Gilles Marckmann. Inflation of elastomeric circular membranes using network constitutive equations. International Journal of Non-Linear Mechanics, 2003, 38 (8), pp.1221-1235. 10.1016/S0020-7462(02)00069-0 . hal-01006966

\section{HAL Id: hal-01006966 https://hal.science/hal-01006966}

Submitted on 6 Oct 2016

HAL is a multi-disciplinary open access archive for the deposit and dissemination of scientific research documents, whether they are published or not. The documents may come from teaching and research institutions in France or abroad, or from public or private research centers.
L'archive ouverte pluridisciplinaire HAL, est destinée au dépôt et à la diffusion de documents scientifiques de niveau recherche, publiés ou non, émanant des établissements d'enseignement et de recherche français ou étrangers, des laboratoires publics ou privés. 


\title{
Inflation of elastomeric circular membranes using network constitutive equations
}

\author{
Verron E. * and Marckmann G. \\ Laboratoire de Mécanique et Matériaux, Division Structures \\ École Centrale de Nantes, BP 92101, 44321 Nantes cedex 3, France
}

Tel: (33) 2.40.37.25.14 Fax: (33) 2.40.37.25.73

\begin{abstract}
The present paper deals with the use of network-based hyperelastic constitutive equations in the context of thin membranes inflation. The study focus on the inflation of plane circular membranes and the materials are assumed to obey Gaussian and non-Gaussian statistical chains network models. The governing equations of the inflation of axisymmetric thin rubber-like membranes are briefly recalled. The material models are implemented in a numerical tool that incorporates an efficient B-spline interpolation method and a coupled Newton-Raphson/arc-length solving algorithm. Two numerical examples are studied: the homogeneous inflation of spherical balloons and the inflation of initially plane circular membranes. In the second example, the inflation profiles and the distributions of extension ratios along the membrane are extensively analyzed during the inflation process. Both examples highlight the need of an accurate modeling of the strain-hardening phenomenon in elastomers.
\end{abstract}

\footnotetext{
* To whom correspondence should be addressed

Email address: erwan.verron@ec-nantes.fr (Verron E.).
} 


\section{Introduction}

In the last few years, classical phenomenological constitutive equations for rubber-like solids, such as Mooney-Rivlin or Ogden models, are progressively replaced by more physical models based on statistical considerations, in various engineering applications [1]. The determination of the material parameters of the constitutive models is often performed using classical homogeneous strain experiments (uniaxial extension or pure shear tests for example). For biaxial deformation, authors use frequently the bubble inflation technique, that consists in inflating an initially plane circular thin membrane (see [16,22] and more recently $[8,20])$. In this type of experiments, deformations are not homogeneous and the analysis of experimental data needs efficient numerical method to solve the inflation problem. The problem of circular membranes inflation is classically solved for phenomenological rubber-like models. Here, we present some solutions corresponding with new statistical constitutive equations.

The governing equations of the large strains inflation of axisymmetric membranes were established by Green and Adkins [11]. Then, some authors solve the corresponding problem by directly integrating the system of ordinary differential equations using shooting methods. Both hyperelastic $[17,33]$ and vis-

coelastic $[30,10]$ constitutive equations are considered. Nevertheless, most of the works are based on numerical discretized methods, such as finite element analysis $[19,5,15,28]$. Most of the papers focus on the numerical methods and consider simple phenomenological constitutive equations. Very recently, Hassager et al. study the response of polymeric fluid membranes under inflation using the advanced Doi-Edwards and Tom-Pom models [12]. 
The aim of the present paper is the analysis of the inflation of thin elastomeric membranes using network based rubber-like constitutive equations. In Section 2, the governing equations of the axisymmetric inflation problem are recalled and the constitutive equations are presented. Four models are studied: the classical neo-Hookean model based on Gaussian statistics and three chains models that are based on non-Gaussian statistics. Section 3 is devoted to the solving procedure. The numerical scheme and our original B-spline interpolation, detailed in [29], are briefly recalled. The numerical analysis of spherical balloons and circular plane membranes are presented in Section 4. Finally, concluding remarks are proposed in Section 5.

\section{Problem formulation}

\subsection{Governing equations}

We consider an axially symmetric hyperelastic membrane of uniform thickness $h_{0}$ in its undeformed configuration. The geometry of this membrane can be described by the cylindrical coordinates of its mid-surface. In each $\theta$-constant plane, both undeformed and deformed configurations are reduced to one-dimensional curves, as shown in Figure 1. Consider a particle of undeformed coordinates $\left(r_{0}, z_{0}\right)$ of the undeformed membrane. This point is displaced to a new position $(r, z)$ in the deformed configuration. The thickness of the deformed membrane is denoted $h$. Every geometrical data $r_{0}, z_{0}, r, z$ and $h$ are functions of the arc-length coordinate in the undeformed configuration $s$, that varies between 0 and $l_{0}$, the length of the initial membrane. As shown by Yang and Feng [33], the principal stretch ratios in meridian, circumferential 
and normal directions are given by:

$$
\lambda_{m}=\sqrt{\frac{r_{, s}^{2}+z_{, s}^{2}}{r_{0, s}^{2}+z_{0, s}^{2}}} \quad, \quad \lambda_{c}=\frac{r}{r_{0}} \quad, \quad \lambda_{n}=\frac{h}{h_{0}}
$$

in which ${ }_{, s}$ stands for the differentiation with respect to $s$. As the material is assumed incompressible, the thickness of the deformed body is simply related to the thickness in the natural state by:

$$
h=\frac{1}{\lambda_{m} \lambda_{c}} h_{0}
$$

Under a quasi-static pressure load, the Principle of Virtual Work can be written in the following form:

$$
\int_{V_{0}} \delta W d V_{0}-\int_{S} \delta \mathbf{u} P \mathbf{n} d S=0 \quad \forall \delta \mathbf{u}
$$

where $V_{0}$ and $S$ are respectively the volume of the undeformed membrane and the surface of the deformed membrane. $W$ is the strain energy, $P \mathbf{n}$ is the outward oriented blowing pressure and $\delta \mathbf{u}$ is an admissible virtual displacement vector. Noting that the components of $\mathbf{n}$ are $\left(z_{, s} / \sqrt{r_{, s}^{2}+z_{, s}^{2}} ;-r_{, s} / \sqrt{r_{, s}^{2}+z_{, s}^{2}}\right)$, the previous equation (3) simplifies:

$$
\int_{0}^{l_{0}} 2 \pi r_{0} h_{0}\left(t_{m} \delta \lambda_{m}+t_{c} \delta \lambda_{c}\right) d s-\int_{0}^{l_{0}} 2 \pi \operatorname{Pr}\left(\delta u z_{, s}-\delta v r_{, s}\right) d s=0
$$

where $t_{m}$ and $t_{c}$ are the principal first Piola-Kirchhoff stresses respectively in the meridian and circumferential directions, and $(\delta u, \delta v)$ stand for the components of the virtual displacement vector.

\subsection{Network constitutive equations for elastomers}

As mentioned above, the use of statistical network models for rubber-like models increases in finite elements tools. These constitutive equations present 
some major advantages: they necessitate a small number of parameters and they agree well with experiments in various modes of deformation [32].

In the statistical approach, polymers are considered as networks of long flexible chains randomly oriented and joined together by chemical cross-links. More details on this subject can be found in $[26,9]$.

\subsubsection{Gaussian statistics model}

Treloar first proposed a statistical treatment of rubber elasticity. He considered that the configuration of polymer chains can be described by Gaussian statistics. This leads to the well-known neo-Hookean constitutive equation [25]. The corresponding strain energy function is:

$$
W=\frac{1}{2} n k T\left(I_{1}-3\right)
$$

where $n$ denotes the average number of polymer chains per unit of volume, $k$ is the Boltzmann constant, $T$ is the absolute temperature and finally $I_{1}$ is the first invariant of the stretch tensor, said its trace. This model only depends on a unique parameter $C_{R}$ defined as:

$$
C_{R}=n k T
$$

\subsubsection{Non-Gaussian statistics models}

In order to overcome the limitations of the previous model that is restricted to small strains, authors use non-Gaussian statistics theory to describe molecular polymer chains configurations. 
2.2.2.1 Single chain elasticity In 1942, Kuhn and Grün use the nonGaussian statistics theory to model the limited extension of chains [18]. Their approach is based on the random walk statistics of an ideal phantom chain.

Consider a molecular chain composed by $N$ monomer segments of length $l$. Its average unstretched length is $\sqrt{N} l$ and its maximum stretched length is $N l$. Moreover, the strain energy function of the chain $w$ can be written in the following form:

$$
w=N k T\left[\frac{\lambda}{\sqrt{N}} \beta+\ln \frac{\beta}{\sinh \beta}\right]
$$

where $\lambda$ is the extension of the chain and with $\beta=\mathcal{L}^{-1}(\lambda / \sqrt{N}) . \mathcal{L}^{-1}$ is the inverse of the Langevin function defined by $\mathcal{L}(x)=\operatorname{coth}(x)-1 / x$. The first Piola-Kirchhoff stress in this single chain is obtained by derivation of the strain energy with respect to the extension:

$$
t=\frac{\partial w}{\partial \lambda}=k T \sqrt{N} \mathcal{L}^{-1}\left(\frac{\lambda}{\sqrt{N}}\right)
$$

2.2.2.2 $p$-chains models In order to develop constitutive equations, a network of chains which strain energy functions are given by (7) should be considered. Recently, Wu and van der Giessen integrate the previous stress (8) on a unit sphere defined by its chains density [32]. Their full network model agrees well with experiments but necessitates numerical integration procedures on the sphere. This numerical integration does not lead to an efficient implementation in numerical softwares.

Other authors used the non-Gaussian statistics theory to develop simpler models which do not need numerical integration. The most employed are the 3chains and the 8-chains models. Moreover, we mention the approached full network model, that accurately approximates the general theory of Wu and 
van der Giessen presented above. These constitutive equations are developed by considering privileged directions in the unit sphere. Denoting $n$ the density of polymer chains as introduced for the neo-Hookean model, it is assumed that $n / p$ chains per unit of volume are oriented in each of the $p$ privileged directions in the undeformed configuration. Then, the previous numerical integration of the strain energy on the unit sphere is advantageously replaced by a sum of $p$ single chain strain energy functions weighted by the factor $n / p$. Particular cases of $p$-chains models are presented in Figure 2.

The simplest $p$-chains model is defined by considering the three principal strain axes as privileged directions. It was derived by James and Guth [13] and is widely known as the 3-chains model. Figure 2(a) presents this model. Principal stresses $\left(t_{i}^{3-c h a i n s}\right)_{i=1,3}$ are expressed as functions of principal stretch ratios $\left(\lambda_{i}\right)_{i=1,3}$ by:

$$
t_{i}^{3 \text {-chains }}=-\frac{q}{\lambda_{i}}+\frac{1}{3} C_{R} \sqrt{N} \mathcal{L}^{-1}\left(\frac{\lambda_{i}}{\sqrt{N}}\right)
$$

where $q$ is the hydrostatic pressure introduced by the incompressibility assumption.

A more recent model based on non-Gaussian statistics is the 8-chains model developed by Arruda and Boyce [2]. Privileged directions are defined by the half diagonals of a cube contained in the unit sphere. Figure 2(b) shows this chains distribution of the model. Its major property is its symmetry with respect to the three principal axes. Therefore, the eight chains are stretched with the same extension ratio, $\lambda^{c h}$, that corresponds to the stretching of each half diagonals of the cube:

$$
\lambda^{\mathrm{ch}}=\sqrt{\left(\lambda_{1}^{2}+\lambda_{2}^{2}+\lambda_{3}^{2}\right) / 3}
$$


This leads to a simple stress-stretch relationship:

$$
t_{i}^{8 \text {-chains }}=-\frac{q}{\lambda_{i}}+\frac{1}{3} C_{R} \sqrt{N} \frac{\lambda_{i}}{\lambda^{\text {ch }}} \mathcal{L}^{-1}\left(\frac{\lambda^{\text {ch }}}{\sqrt{N}}\right)
$$

Finally, we can mention the approached full network model proposed by $\mathrm{Wu}$ and van der Giessen [31]. Authors use a linear combination of the stresses of the 3-chains and 8-chains models to approximate the full network stresses by:

$$
t_{i}^{\text {full network }}=(1-\rho) t_{i}^{3 \text {-chains }}+\rho t_{i}^{8 \text {-chains }}
$$

where $\rho$ is a parameter that is related to the maximal principal stretch ratio:

$$
\rho=\frac{0.85}{\sqrt{N}} \max \left(\lambda_{1}, \lambda_{2}, \lambda_{3}\right)
$$

in which the factor 0.85 is chosen to give the best correlation with the full integration on the unit sphere.

\section{Solution procedure}

In this part, the resolution procedure used to integrate the problem (4) associated with one of the stress-strain relationships defined above is briefly presented. For more details, the reader can refer to [29].

\subsection{Discretized equations and solution procedure}

Whatever the interpolation method adopted, the membrane is discretized by considering $n+1$ nodes, $\left(N_{i}\right)_{i=0, n}$. Each node $N_{i}$ is defined by its undeformed arc-length coordinate $s_{i}$. Its displacements in radial and axial direction are respectively $u_{i}$ and $v_{i}$. The assembled nodal displacements vector, that includes 
all nodal displacements, is denoted $\mathbf{U}$ with:

$$
U_{2 i-1}=u_{i} \text { and } U_{2 i}=v_{i} \quad \forall i=0, \ldots, n
$$

Using interpolation formulas (detailed in the next paragraph), the Principle of Virtual Work (4) becomes:

$$
\delta \mathbf{U}^{T}\left[\mathbf{F}_{\text {int }}(\mathbf{U})-\mathbf{F}_{\text {ext }}(\mathbf{U}, p)\right]=0 \quad \forall \delta \mathbf{U}^{T}
$$

where $\mathbf{F}_{\text {int }}(\mathbf{U})$ and $\mathbf{F}_{\text {ext }}(\mathbf{U}, p)$ are the internal and external forces vectors respectively, $\delta \mathbf{U}$ is the virtual nodal displacement vector and ${ }^{T}$ denotes the transposition. $\mathbf{F}_{\text {int }}$ is the discretized counterpart of the first left-hand side term in Eq. (4) and $\mathbf{F}_{\text {ext }}$ corresponds to the second term in this equation. It is obvious that the system (15) is highly non-linear both geometrically and by the constitutive equation. Thus, an iterative method must be employed to solve this system and the tangent operator must be derived. This operator is denoted $\mathbf{K}$ and represents the derivative of the out-of-balance force vector $\left(\mathbf{F}_{\text {int }}-\mathbf{F}_{\text {ext }}\right)$ with respect to $\mathbf{U}$ :

$$
\mathbf{K}=\frac{\partial \mathbf{F}_{\text {int }}}{\partial \mathbf{U}}-\frac{\partial \mathbf{F}_{\text {ext }}}{\partial \mathbf{U}}
$$

The detailed ready-to-program formulas of $\mathbf{F}_{\text {int }}, \mathbf{F}_{\text {ext }}$ and $\mathbf{K}$ are given in [23] for the classical two nodes membrane finite element and in [29] for our original B-spline interpolation.

As shown by Beatty for static inflation [4] and Verron et al. for dynamic inflation [27], the pressure-displacement curves corresponding to rubber-like membranes inflation exhibit limit points. Consequently a continuation method has to be adopted to compute the equilibrium path [24]. Here, the arc-length method is implemented. It consists in completing the system of equilibrium equations with an additional relation between the load (here the pressure) and 
the displacement increments (see [6] for various versions of this method). In the present case, the displacement control method is considered [3] and the additional equation is:

$$
\|\Delta \mathbf{U}\|^{2}-d a^{2}=0
$$

where $\Delta \mathbf{U}$ is the displacement increment vector between two equilibrium points and $d a$ is the prescribed arc-length. This equation is added to the previous system (15) as a bordered equation and the assembled system is solved by the classical Newton-Raphson algorithm [21].

\subsection{B-splines interpolation method}

In this study, the membrane is interpolated by two cubic splines defined on the set of nodes $\left(N_{i}\right)_{i=0, n}$, one for each coordinate $r_{0}$ and $z_{0}$ :

$$
\begin{aligned}
& r_{0}(s)=\sum_{i=-1}^{n+1} \alpha_{i} B_{i}\left(s / l_{0}\right) \\
& z_{0}(s)=\sum_{i=-1}^{n+1} \beta_{i} B_{i}\left(s / l_{0}\right)
\end{aligned}
$$

where $\left(\alpha_{i}\right)_{i=-1, n+1}$ and $\left(\beta_{i}\right)_{i=-1, n+1}$ are the parameters of the two splines and functions $B_{i}$ are B-spline functions. Denoting $\xi$ the reduced arc-length, said 
$s / l_{0}$, these functions are given by [7]:

$B_{i}(\xi)= \begin{cases}0 & \xi \leq \xi_{i-2} \\ \frac{\left(\xi-\xi_{i-2}\right)^{3}}{\left(\xi_{i+1}-\xi_{i-2}\right)\left(\xi_{i}-\xi_{i-2}\right)\left(\xi_{i-1}-\xi_{i-2}\right)} & \xi_{i-2} \leq \xi<\xi_{i-1} \\ \frac{\left(\xi-\xi_{i-2}\right)^{2}\left(\xi_{i}-\xi\right)}{\left(\xi_{i+1}-\xi_{i-2}\right)\left(\xi_{i}-\xi_{i-2}\right)\left(\xi_{i}-\xi_{i-1}\right)}+\frac{\left(\xi_{i+1}-\xi\right)\left(\xi-\xi_{i-1}\right)\left(\xi-\xi_{i-2}\right)}{\left(\xi_{i+1}-\xi_{i-1}\right)\left(\xi_{i}-\xi_{i-1}\right)\left(\xi_{i+1}-\xi_{i-2}\right)} & \xi_{i-1} \leq \xi<\xi_{i} \\ \frac{\left(\xi_{i+1}-\xi\right)^{2}\left(\xi-\xi_{i-2}\right)}{\left(\xi_{i+1}-\xi_{i-2}\right)\left(\xi_{i+1}-\xi_{i-1}\right)\left(\xi_{i+1}-\xi_{i}\right)}+\frac{\left(\xi-\xi_{i-1}\right)^{2}\left(\xi_{i+2}-\xi\right)}{\left(\xi_{i+1}-\xi_{i-2}\right)\left(\xi_{i}-\xi_{i-1}\right)\left(\xi_{i+2}-\xi_{i-1}\right)} & \\ \frac{\left(\xi_{i+2}-\xi\right)\left(\xi_{i+1}-\xi\right)\left(\xi-\xi_{i-1}\right)}{\left(\xi_{i-1}\right)\left(\xi_{i+1}-\xi_{i-1}\right)\left(\xi_{i+1}-\xi_{i}\right)} & \xi_{i} \leq \xi<\xi_{i+1} \\ \frac{\left(\xi_{i+2}-\xi\right)^{2}\left(\xi-\xi_{i}\right)}{\left(\xi_{i+2}-\xi_{i-1}\right)\left(\xi_{i+2}-\xi_{i}\right)\left(\xi_{i+1}-\xi_{i}\right)} & \\ \frac{}{\left(\xi_{i+2}-\xi_{i-1}\right)\left(\xi_{i+2}-\xi_{i}\right)\left(\xi_{i+2}-\xi_{i+1}\right)} & \xi_{i+1} \leq \xi<\xi_{i+2} \\ 0 & \end{cases}$

where $\xi_{i}$ is the reduced arc-length coordinate of the node $N_{i}$ and where the following conventions are adopted:

$$
\begin{aligned}
& \xi_{i}=s_{0} / l_{0} \text { for } i \leq 0 \\
& \xi_{i}=s_{n} / l_{0} \text { for } i \geq n
\end{aligned}
$$

With these two conventions some denominators are equal to 0 and we adopt the equality: $0 / 0=0$.

In Eqs $(18,19)$, the $n+3$ parameters of each splines are defined in a unique manner using the undeformed coordinates of the $n+1$ nodes and by taking into account end conditions to determine the two extra parameters. Depending on the mechanical boundary conditions, different mathematical end conditions 
for the splines can be adopted:

1. if the extreme point does not lies in the symmetry axis, both $r_{0}$ and $z_{0}$ interpolation admit natural end conditions, i.e. the second derivative of both functions are equal to zero at this point,

2. if the extreme point lies in the symmetry axis, the $z_{0}$-spline admits natural end conditions but the $r_{0}$-spline admits Hermite end conditions, i.e. the first derivative of the function is prescribed at the point and, in our case, is set to zero.

These boundary conditions provide two additional linear relations between parameters for both splines, and interpolation formulas $(18,19)$ reduce to:

$$
\begin{aligned}
& r_{0}(s)=\sum_{i=0}^{n} \alpha_{i} B_{r_{i}}\left(s / l_{0}\right) \\
& z_{0}(s)=\sum_{i=0}^{n} \beta_{i} B_{z_{i}}\left(s / l_{0}\right)
\end{aligned}
$$

where $\left(B_{r_{i}}\right)_{i=0, n}$ and $\left(B_{z_{i}}\right)_{i=0, n}$ are the new interpolation basis, defined as linear combinations of the classical B-spline functions $B_{i}$.

Similarly to isoparametrical finite elements, the displacement field $(u, v)$ is interpolated by two splines developed on the same basis functions:

$$
\begin{aligned}
& u(s)=\sum_{i=0}^{n} \gamma_{i} B_{r_{i}}\left(s / l_{0}\right) \\
& v(s)=\sum_{i=0}^{n} \delta_{i} B_{z_{i}}\left(s / l_{0}\right)
\end{aligned}
$$

in which $\left(\gamma_{i}\right)_{i=0, n}$ and $\left(\delta_{i}\right)_{i=0, n}$ are the parameters of these two splines.

Some calculations are now necessary to establish the discretized problem $(15,16)$. This derivation is detailed in [29]. 


\section{$4 \quad$ Numerical examples}

In this section, some numerical examples are presented.

The aim of this paper is the comparison of different network models. Thus, the different constitutive equations have to be fitted for the same material using the same type of experimental data. Here, experimental data corresponding to the uniaxial extension of a natural rubber as reported by James et al. are considered [14]. Moreover, we adopt the material parameters previously determined by Wu and van der Giessen [32]. It is to note that network parameters $N$ and $C_{R}$ must be determined for each model. But, as the small strain behaviour is governed primarily by $C_{R}$, Wu and van der Giessen first determine it, and consider an identical value of $C_{R}$ for all models, said $C_{R}=0.4 \mathrm{MPa}$. Then afterwards, the average number of monomers per chain is fitted for each constitutive equation: $N=80$ for the 3 -chains model, $N=25$ for the 8 -chains model, and finally $N=50$ for the approached full network model.

\subsection{Spherical balloons}

Before examining the case of circular membranes, we consider the simple homogeneous inflation of spherical balloons. This problem was widely studied for classical hyperelastic models, both in statics [4] and dynamics [27]. As the deformation remains biaxial in every material points of the sphere, the problem reduces to a simple analytical governing equation that relies the blowing pressure and the deformed radius. Here, our numerical approach is used to solve the problem and the numerical results were successfully compared with the analytical relation, that validates the present method. 
Consider a thin spherical membrane of natural rubber. The initial radius is equal to 1.0 and the uniform thickness is 0.01 . The semi-spherical cap is discretized using 21 nodes. It is assumed that the balloon remains spherical during the inflation, so that no bifurcating phenomenon occurs. Consequently, the extension ratios are:

$$
\lambda_{m}=\lambda \quad, \quad \lambda_{c}=\lambda \quad, \quad \lambda_{c}=\frac{1}{\lambda^{2}}
$$

where $\lambda$ is the adimensional deformed radius $r / r_{0}$. The curves pressure versus $\lambda$ are presented in Figure 3 for the four constitutive equations studied here. Values of extension ratios and blowing pressures that correspond to the two limit points, $\left(\lambda_{I}, P_{I}\right)$ and $\left(\lambda_{I I}, P_{I I}\right)$, are presented in Table 1. As shown elsewhere, the neo-Hookean balloon exhibits only one limit point that divides the curve into a stable path and an unstable path. For the three other balloons, the curves admit two limit points and three paths, two stable (increasing parts of the curves) and one unstable (decreasing parts of the curves). A similar behaviour was highlighted previously for the Mooney-Rivlin model [27]. Let us examine more precisely the first part of the curves that correspond to small strains, i.e. for $\lambda \leq 2.0$. First limit points occur approximately for the same values of the extension ratio, said $\lambda=1.39$. This value can be obtained analytically in the case of the neo-Hookean model and is equal to $\sqrt[6]{7}$. This result verifies our previous statement that the small strains behaviour only depends on the material parameter $C_{R}$, that is identical for the four models. For larger strains, inflation curves differ. In the case of the neo-Hookean material, there is no strain-hardening (because of the Gaussian assumption) and the pressure slowly decreases to zero as the membrane continues to inflate. For the three other models, polymer chains reach their limit of extensibility for different values of the undeformed radius. Last points (circles) of the curves in Fig. 3 
represent the last configurations in which the simulation was able to converge. The limit values of the adimensional radius, i.e. values for which the limiting stretch of chains is reached, can be simply predicted for both 3-chains and 8-chains models. In the case of the 3-chains model, the limit of extensibility is reached when one of the extension ratios $\lambda_{i}$ tends to $\sqrt{N}$ in Eq. (9). Here, recalling the equibiaxial deformations (27), we have:

$$
\lambda_{1}=\lambda_{m}=\lambda \quad, \quad \lambda_{2}=\lambda_{c}=\lambda \quad, \quad \lambda_{3}=\lambda_{n}=1 / \lambda^{2}
$$

Thus the limiting extensibility is obtained for $\lambda=\sqrt{80} \approx 8.94$. Note that in our numerical simulations, we are not able to overcome $\lambda \approx 8.0$ due to convergence problems. In the case of the 8-chains model, the stretching of the chains $\lambda^{\text {ch }}$ is defined by Eq. (10). Using (28), it reduces to:

$$
\lambda^{\mathrm{ch}}=\sqrt{\left(2 \lambda^{2}+1 / \lambda^{4}\right) / 3}
$$

and the limit chain extension is obtained by:

$$
\lambda^{\mathrm{ch}}=\sqrt{N}
$$

with $N=25$. The solution of this equation is $\lambda \approx 6.12$. This value can be seen as the asymptotic vertical line corresponding to the pressure curve of the 8-chains model in Fig. 3. Finally, the response of the approached full network model is considered. As the model is a linear combination of the 3-chains and the 8-chains models, the model reaches its limiting extensibility when one of them reaches its own limit. Thus, considering now that $N=50$, the limiting extensibility is obtained for $\lambda=\sqrt{50} \approx 7.07$ for the 3-chains model. For the 8-chains model, Eqs (29) and (30) are solved with $N=50$ and the limiting extensibility is approximately equal to 8.66. The stresses (12) tends to infinity for the smallest value, said $\lambda \approx 7.07$ which corresponds to the 3 -chains stress, 
as shown by the corresponding curve in Fig. 3.

\subsection{Circular plane membranes}

Consider now an initially plane circular membrane of radius 1.0 and uniform thickness 0.01 . The exterior edge of the membrane is clamped. The membrane is meshed using 21 nodes. In this problem, the deformation is not homogeneous in the membrane. At the pole (the node in which $r=0$.), the deformation is equibiaxial:

$$
\lambda_{m} \quad, \quad \lambda_{c}=\lambda_{m} \quad, \quad \lambda_{n}=\frac{1}{\lambda_{m}^{2}}
$$

and at the clamped rim, the membrane is under pure shear conditions:

$$
\lambda_{m} \quad, \quad \lambda_{c}=1 \quad, \quad \lambda_{n}=\frac{1}{\lambda_{m}}
$$

Moreover, it is obvious that the pole lies in the symmetry axis. With our numerical approach, there is no difficulty to satisfy the tangency condition, said $r^{\prime}=0$, because this boundary condition is directly taken into account in the $B_{r_{i}}$ interpolation functions (see Section 3.2). Using classical finite elements, this condition can not be strictly imposed, because there is no degree of freedom that corresponds to $r^{\prime}$ (see [12] for example).

First, we study the curves that show the inflating pressure versus the axial coordinate of the pole (denoted $z_{\text {pole }}$ through the rest of the paper) for the different constitutive equations. These results are shown in Figure 4. The corresponding values of limit points $\left(z_{\text {pole } I}, P_{I}\right)$ and $\left(z_{\text {pole } I I}, P_{I I}\right)$ are presented in Table 2. General aspects of the curves are similar to those observed for the spherical balloons with both increasing and decreasing paths. In the present case, the two values of the pressure that correspond to limit points are not very 
different for each chains models. Moreover, the strain-hardening of the nonGaussian constitutive equations is highly remarkable and the limiting chain extension is rapidly reached as it can be shown by the final vertical parts of curves presented in Fig. 4.

Let us now examine the inflation profiles shown in Figure 5. For the four models, we present profiles that correspond to $z_{\text {pole }}=1.0,2.0,3.0$ and 4.0. Moreover, the last profiles obtained before numerical divergence are also drawn for the non-Gaussian constitutive equations. It can be seen that for $z_{\text {pole }}$ equal to 1.0 and 2.0 the profiles corresponding to the four models are similar. Nevertheless, for higher values of the axial coordinate of the pole, said $z_{\text {pole }} \geq 3.0$, the profiles corresponding to the three non-Gaussian models are similar, but they highly differ from the shape of the deformed neo-Hookean membranes. The neo-Hookean bubble is more "spherical" than the other ones. This example shows that the strain-hardening of the material has a very significant influence of the shape of inflated membranes.

This remark is confirmed by the thickness distributions presented in Figure 6 . The thickness is plotted as function of the undeformed radius $r_{0}$. As observed by Hassager et al., the neo-Hookean membrane exhibits a strong thinning at the pole [12]. Here, we show that the same observations can be made for nonGaussian statistical models. As predicted by the examination of the profiles, the three non-Gaussian thickness distributions are almost similar at the pole, and are of the same order of magnitude along the membrane for a given value of $z_{\text {pole }}$. The last profile of each non-Gaussian membrane exhibits the same thickness distribution that can be seen as the ultimate state before the occurrence of chains rupture. Moreover, the difference between the neo-Hookean membrane and the non-Gaussian membranes is very significant. For values of 
$z_{\text {pole }}$ of $2.0,3.0$ and 4.0, the thickness of the neo-Hookean membrane at the clamped rim are identical and values at the pole tend to zero (see Fig. 6(a)). In the case of the three other models, the thickness at the clamped rim evolves significantly with respect to $z_{\text {pole }}$ and the thinning at the pole remains smaller than in the neo-Hookean case.

In order to highlight the influence of the strain-hardening modeled by the inverse Langevin function in constitutive equations (9), (11) and (12), the distribution of the in-plane extension ratios $\lambda_{m}$ and $\lambda_{c}$ along the membranes are presented in Figure 7. First, we verify that the circumferential stretch ratio $\lambda_{c}$ varies between $\lambda_{m}$ at the pole and 1.0 at the clamped rim. Fig. 7(a) reveals that, for the neo-Hookean bubble, both distributions are similar, as previously demonstrated by Yang and Feng [33]. Moreover, for $z_{\text {pole }}=5.0$, extensions ratios are equal to 25.0 at the pole. This result has no physical meaning and proves the importance of a good modeling of strain-hardening of elastomers at large strains. In the case of the non-Gaussian models, distributions of $\lambda_{c}$ for the three models are comparable for the same deformation level. Distributions of $\lambda_{m}$ are also similar until the material reaches its strain-hardening behaviour. In this case, the 8-chains models distribution (Fig. 7(c) for $z_{\text {pole }}=4.02$ ) differs from the 3-chains and the approached full network distributions (Fig. 7(b) for $z_{\text {pole }}=5.60$ and Fig. $7(\mathrm{~d})$ for $\left.z_{\text {pole }}=3.87\right)$. In the former case, $\lambda_{m}$ is greater at the clamped extremity than at the pole; in the later, $\lambda_{m}$ is approximately uniform for both 3-chains and approached full network membranes. This last observation can be explained by examining the maximum chain extensibility along the membrane for the three non-Gaussian bubbles. Recall that for the 3-chains model, the maximum chain extension at a material point is defined 
by:

$$
\lambda^{\text {ch } \max }=\max \left(\lambda_{m}, \lambda_{c}, \lambda_{n}\right)
$$

and in our case, it is always equal to $\lambda_{m}$. For the 8-chains model, it is given by:

$$
\lambda^{\mathrm{ch} \max }=\sqrt{\left(\lambda_{m}^{2}+\lambda_{c}^{2}+\lambda_{n}^{2}\right) / 3}
$$

and finally, for the approached full network model, it is the greater values of the 3-chains and 8-chains data:

$$
\lambda^{\text {ch } \max }=\max \left(\lambda_{m}, \lambda_{c}, \lambda_{n}, \sqrt{\left(\lambda_{m}^{2}+\lambda_{c}^{2}+\lambda_{n}^{2}\right) / 3}\right)
$$

and here, it is always equal to $\lambda_{m}$. In the three cases, as $\lambda^{\text {ch max }}$ tends to $\sqrt{N}$, the material hardens and the asymptotic vertical line in the pressure versus $z_{\text {pole }}$ diagram is approached (Fig. 4). The distributions of $\lambda^{\text {ch max }}$ along the membranes are plotted versus the undeformed radius in Figure 8, for the three non-Gaussian network models. They are highly similar for the three models: at the beginning of the inflation the distribution is uniform, then the membrane extends more significantly in the neighbourhood of the pole than near the clamped rim, and finally for higher values of $z_{\text {pole }}$ the chain extensibility tends to become uniform and approaches the limit value $\sqrt{N}$, while few evolution of strains takes place at the pole. This can be seen as the inflation of a circular membrane with a rigid inclusion at the pole, considering that the radius of this inclusion increases during the inflation.

\section{Concluding remarks}

In this paper, we have presented some results concerning the inflation of rubber-like circular membranes using physical networks hyperelastic consti- 
tutive equations. The simulations are performed using a new B-spline model that was proved to be very efficient for large strains inflation of axisymmetric membranes. The non-Gaussian models are implemented and compared with the classical neo-Hookean constitutive equation. The influence of strainhardening on the membrane response is highlighted. It is shown that both inflation profiles and thickness distribution are highly influenced by the nature of the material model.

These results are of interest in the context of the identification of material parameters in biaxial conditions. In order to perform biaxial experiments on elastomers or heat-softened plastics, the bubble inflation technique is widely used. In most of the cases, only experimental data obtained at the pole are considered to determine parameters of constitutive equations, because the deformation is equibiaxial at this point. The present work shows that the evolution of the membrane profile during inflation and some data concerning the thickness distribution may be of great interest in the choice of the constitutive equation that must be adopted, and in the determination of the corresponding material parameters. Thus, the numerical analysis of the entire membrane is highly suitable in the identification procedure.

\section{References}

[1] J. M. Allport and A. J. Day, Statistical mechanics material model for the constitutive modelling of elastomeric compounds. Proc. Instn Mech. Engrs Part C 210, 575 (1996).

[2] E. Arruda and M. C. Boyce, A three-dimensional constitutive model for the large stretch behavior of rubber elastic materials. J. Mech. Phys. Solids 41, 
389 (1993).

[3] J. L. Batoz and G. Dhatt, Incremental displacement algorithms for nonlinear problems. Int. J. Num. Meth. Engng 14, 1262 (1979).

[4] M. F. Beatty, Topics in finite elasticity: hyperelasticity of rubber, elastomers, and biological tissues - with examples. Appl. Mech. Rev. 40, 1699 (1987).

[5] J. M. Charrier, S. Shrivastava and R. Wu, Free and constrained inflation of elastic membranes in relation to thermoforming - Axisymmetric problems. $J$. Strain Analysis 22, 115 (1987).

[6] M. A. Crisfield, Non-linear finite element analysis of solids and structures. Volume 1: Essentials. John Wiley and Sons Ltd, Chichester, England (1994).

[7] C. De Boor, A practical guide to splines. Springer, New-York (1978).

[8] A. Derdouri, F. Erchiqui, A. Bendada, E. Verron and B. Peseux, Viscoelastic behaviour of polymer membranes under inflation. Proceedings of the 13th International Congress on Rheology, Cambridge UK, August 20-25, 3.394 (2000).

[9] M. Doi, Introduction to Polymer Physics. Clarendon Press, Oxford (1996).

[10] W. W. Feng, Viscoelastic behavior of elastomeric membranes, J. Appl. Mech. ASME 59, S29 (1992).

[11] A. E. Green and J. E. Adkins, Large elastic deformations. The Clarendon Press, Oxford (1960).

[12] O. Hassager, S. B. Kristensen, J. R. Larsen and J. Neergaard, Inflation and instability of a polymeric membrane. J. Non-Newtonian Fluid Mech. 88, 185 (1999).

[13] H. M. James and E. Guth, Theory of the elastic properties of rubber, J. Chem. Phys. 11, 455 (1943). 
[14] A. G. James, A. Green and G. M. Simpson, Strain energy functions of rubber. I. Characterization of gum vulcanizates. J. Appl. Polym. Sci. 19, 2033 (1975).

[15] L. Jiang and J. B. Haddow, A finite element formulation for finite static axisymmetric deformation of hyperelastic membranes. Comput. Struct. 57, 401 (1995).

[16] D. D. Joye, G. W. Poehlein and C. D. Denson, A bubble inflation technique for the measurement of viscoelastic properties in equal biaxial flow. Trans. Soc. Rheol. 17, 421 (1972).

[17] W. W. Klingbeil and R. T. Shield, Some numerical investigations on empirical strain energy functions in the large axi-symmetric extensions of rubber membranes. Z. Angew. Math. Phys. 15, 608 (1964).

[18] W. Kuhn and F. Grün, Beziehungen zwichen elastischen konstanten und dehnungsdoppelbrechung hochelastischer stoffe. Kolloideitschrift 101, 248 (1942).

[19] J. T. Oden and T. Sato, Finite strains and displacements of elastic membranes by the finite element method. Int. J. Solids Struct. 3, 471 (1967).

[20] N. Reuge, F. M. Schmidt, Y. Le Maoult, M. Rachick and F. Abbé, Elastomer biaxial characterization using bubble inflation technique. I: Experimental investigations. Polym. Engng Sci. 41, 522 (2001).

[21] E. Riks and C. C. Rankin, An incremental approach to the solution of snapping and buckling problems. Int. J. Solids Struct. 15, 529 (1979).

[22] L. R. Schmidt and Carley J. F., Biaxial stretching of heat-softened plastic sheets: experiments and results. Polym. Engng Sci. 15, 51 (1975).

[23] J. Shi and G. F. Moita, The post-critical analysis of axisymmetric hyper-elastic membranes by the finite element method. Comput. Methods Appl. Mech. Engng 135, 265 (1996). 
[24] T. Sokól and M. Witkowski, Some experiences in the equilibrium path determination. Comput. Assist. Mech. Engng Sci. 4, 189 (1997).

[25] L. R. G. Treloar, The elasticity of a network of long chain molecules (I and II). Trans. Faraday Soc. 39, 36, 241 (1943).

[26] L. R. G. Treloar, Physics of rubber elasticity, 3rd Edition. University Press, Oxford (1975).

[27] E. Verron, R. E. Khayat, A. Derdouri and B. Peseux, Dynamic inflation of hyperelastic spherical membranes. J. Rheol. 43, 1083 (1999).

[28] E. Verron, G. Marckmann and B. Peseux, Dynamic inflation of non-linear elastic and viscoelastic rubberlike membranes. Int. J. Numer. Meth. Engng. 50, 1233 (2001).

[29] E. Verron and G. Marckmann, An axisymmetrical B-splines model for the nonlinear free inflation of rubberlike membranes. Comput. Meth. Appl. Mech. Engng 190, $6271(2001)$.

[30] A. Wineman, On axisymmetric deformations of nonlinear viscoelastic membranes. J. Non-Newtonian Fluid Mech. 4, 249 (1978).

[31] P. D. Wu and E. van der Giessen, On improved 3-D non-gaussian network models for rubber elasticity. Mech. Res. Comm. 19, 427 (1992).

[32] P. D. Wu and E. van der Giessen, On improved network models for rubber elasticity and their applications to orientation hardening in glassy polymers. $J$. Mech. Phys . Solids 41, 427 (1993).

[33] W. H. Yang and W. W. Feng, On axisymmetrical deformations of nonlinear membranes. J. Appl. Mech. ASME 37, 1002 (1970). 


\section{List of captions}

Fig. 1. Problem description.

Fig. 2. Description of the most employed p-chains models: (a) 3-chains, (b) 8-chains.

Fig. 3. Pressure versus adimensional deformed radius for spherical balloons.

Fig. 4. Pressure versus axial coordinate of the pole for the plane membranes.

Fig. 5. Inflation profiles of the circular plane membranes: (a) neo-Hookean, (b) 3-chains, (c) 8-chains, (d) approached full network. Numbers on the curves stand for the corresponding inflating pressure.

Fig. 6. Thickness distribution in the circular plane membranes: (a) neo-Hookean, (b) 3-chains, (c) 8-chains, (d) approached full network. Numbers on the curves stand for the corresponding value of $z_{\text {pole }}$.

Fig. 7. Extension ratios in the circular plane membranes $(-) \lambda_{m},(\cdots) \lambda_{c}$ : (a) neo-Hookean, (b) 3-chains, (c) 8-chains, (d) approached full network. Numbers on the curves stand for the corresponding value of $z_{\text {pole }}$.

Fig. 8. Maximum chains extensibility in the circular plane membranes (-) distribution in the membrane, (- -) limit value $\sqrt{N}$ : (a) 3-chains, (b) 8-chains, (c) approached full network. Numbers on the curves stand for the corresponding value of $z_{\text {pole }}$. 
Tables

\begin{tabular}{|c||c|c||c|c|}
\hline Model & $\lambda_{I}$ & $P_{I}$ & $\lambda_{I I}$ & $P_{I I}$ \\
\hline Neo-hookean & 1.40 & $4.96 \mathrm{e}-03$ & $\cdot$ & $\cdot$ \\
\hline 3-chains & 1.40 & $5.04 \mathrm{e}-03$ & 5.71 & $1.97 \mathrm{e}-03$ \\
\hline 8-chains & 1.39 & $5.13 \mathrm{e}-03$ & 3.92 & $2.88 \mathrm{e}-03$ \\
\hline Approached full network & 1.39 & $5.08 \mathrm{e}-03$ & 5.10 & $2.26 \mathrm{e}-03$ \\
\hline
\end{tabular}

Table 1

Limit points for spherical balloons. Circles represent the analytical solutions.

\begin{tabular}{|c||c|c||c|c|}
\hline Model & $z_{\text {pole } I}$ & $P_{I}$ & $z_{\text {pole } I I}$ & $P_{I I}$ \\
\hline Neo-hookean & 1.16 & $7.52 \mathrm{e}-03$ & $\cdot$ & $\cdot$ \\
\hline 3-chains & 1.23 & $7.73 \mathrm{e}-03$ & 3.16 & $6.09 \mathrm{e}-03$ \\
\hline 8-chains & 1.17 & $8.01 \mathrm{e}-03$ & 2.17 & $7.64 \mathrm{e}-03$ \\
\hline Approached full network & 1.26 & $7.84 \mathrm{e}-03$ & 2.83 & $6.66 \mathrm{e}-03$ \\
\hline
\end{tabular}

Table 2

Limit points for circular plane membranes. 